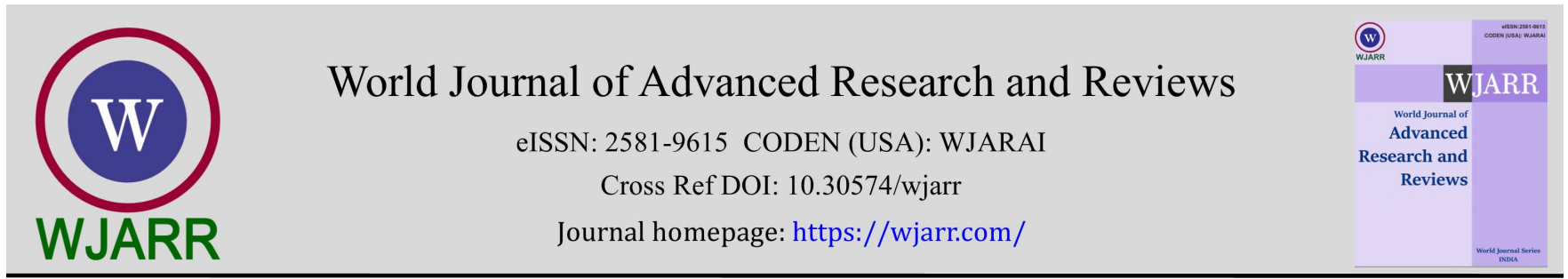

(RESEARCH ARTicle)

Check for updates

\title{
Impact of MDCAT scores on pessimism, loneliness, and depressive symptoms: Moderating role of coping strategies
}

\author{
Iram Fatima 1, Masood Nadeem 2, Muhammad Waqar Naeem ${ }^{3}$ and Hafiz Muhammad Zeeshan Raza 4,* \\ ${ }^{1}$ MS Scholar, Department of Applied Psychology, Islamia University of Bahawalpur, Punjab, Pakistan. \\ 2 Professor, Department of Applied Psychology, Islamia University of Bahawalpur, Punjab, Pakistan. \\ ${ }^{3}$ MS Scholar, Department of Law and Economics, Islamia University of Bahawalpur, Punjab, Pakistan. \\ ${ }^{4}$ Research Associate, Genomics and Computational Biology Laboratory, COMSATS University Islamabad, Sahiwal Campus, \\ Punjab, Pakistan.
}

World Journal of Advanced Research and Reviews, 2021, 12(02), 354-364

Publication history: Received on 09 October 2021; revised on 12 November 2021; accepted on 14 November 2021

Article DOI: https://doi.org/10.30574/wjarr.2021.12.2.0600

\begin{abstract}
This study was designed to evaluate the impact of MDCAT scores on pessimism, loneliness, and depressive symptoms. Furthermore, to check the moderating effect of coping strategies. Purposive sample technique was used to get the relevant sample. MDCAT failures $(\mathrm{N}=149)$ from different educational organization of Bahawalpur (Pakistan) were included in the research as sample. Demographic form, Siddiqui Shah Depression Scale, UCLA Loneliness Scale, Life Orientation Test and Coping Styles Scale were administered to assess the impact of MDCAT scores on pessimism, depressive and loneliness symptoms among MDCAT failures and to assess the moderating role of coping strategies. For statistical analysis PLS and SPSS software were used. PLS algorithm, PLS bootstrapping, PLS moderation analysis and independent sample t-test were used to get the results. Results reveal that there is a significant impact of MDCAT scores on pessimism, depressive and loneliness symptoms. Also, there is significant positive relationship among pessimism, depressive and loneliness symptoms. Problem focused coping significantly moderate the relationship between MDCAT scores and depressive symptoms. Furthermore, problem focused coping has significant negative relationship with pessimism, depressive and loneliness symptoms. Moreover, emotion focused coping has significant positive relationship with depressive symptoms. Female were found having more emotion focused coping. The findings of the study have practical implications in providing insight relevant for development of psychological disturbance in MDCAT failures.
\end{abstract}

Keywords: MDCAT score; Pessimism; Loneliness; Depressive; Medical Students

\section{Introduction}

Everyone has some goals in their life and wants to achieve it. Almost every child has set goal of what he or she want to become in the future [1]. But when they don't get what they want, then psychological distress and problems arise. Failure of goal is very essential to study because it influences on wellbeing and also impact on taking a stab at future objectives. The present study focuses on mental health problems like depressive symptoms, pessimism and loneliness that MDCAT (Medical and Dental College Admission Test) failures face. As well as what coping tactics they use to overcome from that stressful situation [2]. Discoveries demonstrated that people with PD utilized comparable measures of problem-focused coping systems and emotion-focused coping methodologies, and they detailed more optimism than pessimism. There was just a single sexual category distinction: Females revealed more problem-focused coping than males [3]. Optimism was emphatically identified with both emotion-focused and problem-focused coping, and

\footnotetext{
${ }^{*}$ Corresponding author Hafiz Muhammad Zeeshan Raza

Genomics and Computational Biology Laboratory, COMSATS University Islamabad, Sahiwal Campus, Punjab, Pakistan. 
pessimism was adversely identified with emotion-focused and problem-focused coping. Optimism and pessimism completely intervened the connection between coping methodologies and depressive symptoms [4].

Pre medical students have to struggle more because their subject demands so. They face many psychological and academic stressors that can lead to depression [5]. A study conducted by Adhikari (2017) on dysfunctional career thinking as a predictor of hopelessness and depression in students seeking career services. The findings of this study show that all indications of dysfunctional career thinking, despair, and pessimism have a progressive relationship [6]. Additionally, a variety of characteristics of dysfunctional career thinking can be utilised to predict melancholy and pessimism in undergraduate and graduate students seeking career assistance. Persistence in loneliness, start out depression. Someone when get away from family and from his friend even away from social circle or environment then loneliness and depression become a chain reaction [7].

Cognitive coping techniques appeared to play a good greater essential function. Self-blame, catastrophizing, rumination, fine reappraisal, and positive refocus were all linked to depression symptoms [8]. Furthermore, positive reappraisal and self-blame rumination are thought to play a moderating effect in the link between stress levels and depression symptoms. Among undergraduate students decrease depressive ratings had been related to expanded problem-focused coping. Furthermore, with elevated perceived loneliness, advocated decrease problem-focused coping. Findings propose that Val/Val people might also have negative neuro cognitive vulnerability to loneliness reports [9].

After going through a rigorous effort, looking into literature it is believed that, many of psychological issues exist in society and sometime specific issues are related to specific age and specific circumstances. Intermediate is twelve years of education which is decisive time period for the students [10]. Because it is assumed that this is the time that leads students to different professions. Students work hard to get success in terms of got admission in their desired field. Major two groups are there in Pakistan, science and arts. It is evident that society plays very curricle role, because there is lots of prestige, power and respect given to doctors and other science field workers. In Pakistan MDCAT score is becoming a burning issue in students [11]. Every premedical student works with his/her full zeal and zest to be the part of merit. The aim of this study is to investigate student's psychological problems that they are facing due to high merit and their below merit scores. Those students who may have capability to become a doctor but due to some factors might be internal or external, they are unable to compete. Every year thousands of people appear in MDCAT and only few of them got succeeded. In 2018, UHS (University of Health Sciences) reported that 61900 students appear in MDCAT and seats were only three thousand [11].

No research has done so far on the impacts of MDCAT scores yet. And this is becoming a key issue in Pakistan. It should be assessed which variables are connected to psychological health of students like, pessimist, loneliness and depression so these variables can be effected and should be researched on. It is thought that in adjusting to unattainable goals individual differences are susceptibility indicator for depression. Earlier study has found that pessimism in depression prolongs to personal approach and avoidance goals. This combination of variables is also missing in literature and it reveals important and useful aspects about student's psychological health. This research will helpful for professional to understand what problem they are facing and what should treatment apply on them. This study also helpful for the parents to understand the seriousness and brought up their child with flexibilities in their aims of life.

\section{Objectives}

The main objectives of the study were to measure the impact of MDCAT scores on pessimism, loneliness, and depressive symptoms in addition to relationship between them. It also checked the moderating role of emotion and problem focused coping between MDCAT scores and, pessimism, depressive and loneliness symptoms.

\section{Methods}

\subsection{Participant's Characteristics}

Total number of $(N=149$ ) of both gender (male \& female) was included in the study of 60 to $89 \%$ MDCAT failure. Researcher approached to almost 300 MDCAT failures but the response rate was almost 50\%. They were not willing to fill the questionnaires because they were very busy with their hectic studies. And also they don't want to do so because supposedly they were overwhelmed with their depressive tendencies. 


\subsubsection{Inclusion Criteria}

The target population of the study was those candidates that appear in MDCAT in 2018 and have 60 to $89 \%$ scores. The entire sample was selected from lower, middle and upper class.

\subsubsection{Exclusion Criteria}

Those candidates who are below 60\% merit of MDCAT and who are on merit and becoming a doctor was also excluded from the study.

\subsection{Sampling Procedure}

Non probability sampling technique, purposive sampling was used to get relevant participants. Non probability sampling is used when there are known number of elements, having similar characteristics. Moreover, Purposive sampling is used for specific purpose of selection of participants on the basis of their qualities.

\subsection{Sample Size, Power and Precision}

Sample size for the research was calculated from $\mathrm{G}^{*}$ Power. It is a sample size calculator and statistical power computing software for many different tests, F-tests, t-tests, z-tests and exact some tests. Because of initial modal of research was multivariate analysis, as there is one independent variable (MDCAT scores) with three self-constructed levels (high, medium and low scores), three dependent variables (pessimism, depressive and loneliness symptoms), with effect size 0.15 , level of significance 0.05 and statistical power 0.85 . According to $G^{*}$ Power software minimum sample size was 141 within three categories. For current research sample size for high, medium and low, was 49, 50 and 50 respectively according to their responding.

\subsection{Research Design}

When there are more than two dependent variables and independent variable with different levels than a quantitative, cross-sectional and multivariate research design is well suited for this type of studies.

\subsection{Procedure}

Researcher approached to different educational organizations for data collection. Response rate was very low. Scales was used in the study and informed consent also taken from participants. If they agreed, participants were requested to sign a consent form. According to the study's requirements, the researcher delivered the self-developed demographic information sheet (DIS). The demographic information sheet included age, SES, gender, and MDCAT percentages, among other things. Individuals who met the study's inclusion criteria were included in study for the purpose of administering further research questionnaires. The researcher administered the Coping Style Scale, Siddiqui Shah Depression Scale, UCLA Loneliness Scale and Life Orientation Test by implementing same process and setting for administration of all questionnaires.

\subsection{Statistical Analyses}

Analysis of the data was completed by using SPSS-22 and Smart PLS-SEM-3 (Partial Least Square) statistical analysis software, PLS software was used for statistical analysis because first of all it is the latest software for analysis, it incorporates multiple dependent constructs and explicitly recognizes measurement error (Karim, 2009) and this study also incorporates more than one dependent variables, similarly PLS especially useful for prediction of different constructs (Urbach \& Ahleman, 2010) so PLS-SEM was used specifically for data analysis due to all of the above reasons. By using Smart PLS-SEM (Partial Least Square) statistical analysis software, psychometric properties of the filled questionnaires were calculated through factor loadings, likewise path coefficient and t-value was calculated through algorithm and bootstrapping, and $\mathrm{R}^{2}$ coefficient and $\mathrm{Q}^{2}$ coefficient was calculated through blindfolding for measuring the effects of study variables. Statistical package for social sciences SPSS-21 was applied for frequency distribution of demographic variables and independent $t$ testing was used for gender differences.

\subsection{Ethical Considerations}

All the ethics of psychological research were followed. Permission was taken from the authors of all scales. Informed consent was also taken and participants were free to leave the study if they wanted to. Debriefing was also given to participants. One or two unfilled item containing questionnaires was also discarded from research. 


\section{Results}

\subsection{Demographics}

There were 149 overall participants of current study including 62 males and 87 females. They include 129 participants within the age of 17-20 years and 20 within 21-23 years. Total of only two participants were from low class family, 125 from middle class, and 22 from high class family (Table 1).

Table 1 Frequency Distribution of Demographic Variables

\begin{tabular}{|l|c|c|c|}
\hline \multirow{3}{*}{ Gender } & Variables & $\boldsymbol{f}$ & $\mathbf{\%}$ \\
\hline \multirow{3}{*}{ Age } & Male & 62 & 41.6 \\
\cline { 2 - 4 } & Female & 87 & 58.4 \\
\hline \multirow{3}{*}{ Socio Economic Status } & $17-20$ & 129 & 86.5 \\
\cline { 2 - 4 } & $21-23$ & 20 & 13.5 \\
\cline { 2 - 4 } & Low & 2 & 1.3 \\
\cline { 2 - 4 } & Middle & 125 & 83.9 \\
\cline { 2 - 4 } & High & 22 & 14.8 \\
\hline
\end{tabular}

Life orientation on Loneliness and depression Scale

As shown in table 2, the factor loading of all the 4 indicators of the constructs life orientation test, subscale pessimism and 20 indicators of UCLA Loneliness Scale (Russell, 1996) and 36 indicators of Siddiqui shah depression scale (Siddiqui, 1992) were in the range of 0.417 and 0.759 , which is satisfactory and acceptable and if less than 0.4 factor loading than remove that indicator according to Hair et al. (2017). So, two items of SSDS (4 and 12) were removed. In this table Average Variance Estimated (AVE) is 0.430 for pessimism, 0.3933 for loneliness scale and 0.371 for SSDS which is acceptable when composite reliability is more than 0.60 and 0.7 to 0.9 is desirable (Fornell \& Larcker, 1981). Similarly, the discriminant validity of all scales is established through cross-loadings and Fornell-Larcker criterion method, according to which if cross loading is above 0.5 than yes should be given to discriminant validity.

Table 2 Reliability and Validity for Life Orientation Test, UCLA Loneliness Scale and Siddiqui Shah Depression Scale

\begin{tabular}{|c|c|c|c|c|c|c|c|}
\hline $\begin{array}{c}\text { Latent } \\
\text { Variable }\end{array}$ & Indicators & $\begin{array}{c}\text { Factor } \\
\text { Loadings }\end{array}$ & $\begin{array}{c}\text { Indicator } \\
\text { Reliability }\end{array}$ & $\boldsymbol{\alpha}$ & CR & AVE & $\begin{array}{c}\text { Discriminant } \\
\text { Validity }\end{array}$ \\
\hline Life & Pessimism-1 & 0.676 & 0.45 & 0.562 & 0.748 & 0.430 & Yes \\
Orientation & Pessimism-2 & 0.704 & 0.50 & & & & \\
Scale & Pessimism-3 & 0.519 & 0.27 & & & & \\
& Pessimism-4 & 0.705 & 0.50 & & & & \\
\hline UCLA & UCLA-1 & 0.574 & 0.33 & 0.919 & 0.927 & 0.3933 & Yes \\
Loneliness & UCLA-2 & 0.600 & 0.36 & & & & \\
Scale & UCLA-3 & 0.661 & 0.37 & & & & \\
& UCLA-4 & 0.717 & 0.52 & & & & \\
& UCLA-5 & 0.608 & 0.37 & & & & \\
& UCLA-6 & 0.419 & 0.18 & & & & \\
& UCLA-7 & 0.683 & 0.47 & & & & \\
& UCLA-8 & 0.731 & 0.54 & & & & \\
& UCLA-9 & 0.628 & 0.40 & & & & \\
& UCLA-10 & 0.596 & 0.36 & & & & \\
& UCLA-11 & 0.656 & 0.43 & & & & \\
& UCLA-12 & 0.535 & 0.29 & & & & \\
& UCLA-13 & 0.496 & 0.25 & & & \\
\end{tabular}




\begin{tabular}{|c|c|c|c|c|c|c|c|}
\hline & $\begin{array}{l}\text { UCLA-14 } \\
\text { UCLA-15 } \\
\text { UCLA-16 } \\
\text { UCLA-17 } \\
\text { UCLA-18 } \\
\text { UCLA-19 } \\
\text { UCLA-20 }\end{array}$ & $\begin{array}{l}0.640 \\
0.647 \\
0.634 \\
0.612 \\
0.627 \\
0.668 \\
0.721\end{array}$ & $\begin{array}{l}0.41 \\
0.42 \\
0.40 \\
0.38 \\
0.40 \\
0.45 \\
0.52\end{array}$ & & & & \\
\hline $\begin{array}{c}\text { Siddiqui } \\
\text { Shah } \\
\text { Depression } \\
\text { Scale }\end{array}$ & $\begin{array}{c}\text { SSDS-1 } \\
\text { SSDS-2 } \\
\text { SSDS-3 } \\
\text { SSDS-4 } \\
\text { SSDS-5 } \\
\text { SSDS-6 } \\
\text { SSDS-7 } \\
\text { SSDS-8 } \\
\text { SSDS-9 } \\
\text { SSDS-10 } \\
\text { SSDS-11 } \\
\text { SSDS-12 } \\
\text { SSDS-13 } \\
\text { SSDS-14 } \\
\text { SSDS-15 } \\
\text { SSDS-16 } \\
\text { SSDS-17 } \\
\text { SSDS-18 } \\
\text { SSDS-19 } \\
\text { SSDS-20 } \\
\text { SSDS-21 } \\
\text { SSDS-22 } \\
\text { SSDS-23 } \\
\text { SSDS-24 } \\
\text { SSDS-25 } \\
\text { SSDS-26 } \\
\text { SSDS-27 } \\
\text { SSDS-28 } \\
\text { SSDS-29 } \\
\text { SSDS-30 } \\
\text { SSDS-31 } \\
\text { SSDS-32 } \\
\text { SSDS-33 } \\
\text { SSDS-34 } \\
\text { SSDS-35 } \\
\text { SSDS-36 }\end{array}$ & $\begin{array}{l}0.607 \\
0.649 \\
0.687 \\
0.395 \\
0.515 \\
0.626 \\
0.617 \\
0.620 \\
0.417 \\
0.550 \\
0.611 \\
0.165 \\
0.563 \\
0.502 \\
0.679 \\
0.759 \\
0.608 \\
0.661 \\
0.649 \\
0.648 \\
0.604 \\
0.692 \\
0.726 \\
0.553 \\
0.640 \\
0.728 \\
0.684 \\
0.663 \\
0.604 \\
0.457 \\
0.633 \\
0.474 \\
0.719 \\
0.633 \\
0.544 \\
0.670\end{array}$ & $\begin{array}{l}0.45 \\
0.43 \\
0.48 \\
0.16 \\
0.27 \\
0.40 \\
0.38 \\
0.39 \\
0.17 \\
0.30 \\
0.38 \\
0.03 \\
0.32 \\
0.25 \\
0.46 \\
0.58 \\
0.37 \\
0.44 \\
0.43 \\
0.43 \\
0.37 \\
0.48 \\
0.53 \\
0.31 \\
0.41 \\
0.53 \\
0.47 \\
0.44 \\
0.37 \\
0.22 \\
0.40 \\
0.23 \\
0.52 \\
0.40 \\
0.30 \\
0.45\end{array}$ & 0.949 & 0.954 & 0.371 & Yes \\
\hline
\end{tabular}

\subsection{Correlation Outcomes}

The table 3 shows the relationships among depressive symptoms, pessimism and loneliness. According to the above table correlation between depressive symptoms and loneliness is assessed through path coefficient $=0.738$, whereas $\mathrm{t}$ value $=26.353$ and $Q^{2}=0.188$ also shows there is a significant relationship between depressive symptoms and loneliness. Whereas the value of $\mathrm{R}^{2}$ coefficient $=0.545$ shows large effect size of depressive symptoms on loneliness. According to the above table correlation between depressive symptoms and pessimism is assessed through path coefficient $=0.598$, whereas $t$-value $=10.720$ and $Q^{2}=0.139$ also shows there is a significant relationship between depressive symptoms and pessimism. Whereas the value of $R^{2}$ coefficient $=0.357$ shows medium effect size of depressive symptoms on pessimism. Similarly, according to the above table correlation between pessimism and loneliness is assessed through path coefficient $=0.478$, whereas $t$-value $=8.823$ and $Q^{2}=0.074$ also shows there is a significant relationship between pessimism and loneliness. Whereas the value of $R^{2}$ coefficient $=0.229$ shows medium effect size of pessimism on loneliness. 
Table 3 Significance of Path Coefficient for Life Orientation Test, UCLA Loneliness Scale and Siddiqui Shah Depression Scale

\begin{tabular}{|c|c|c|c|c|c|c|c|}
\hline Relationship & Path & t-value & $\mathbf{p}$ & $\mathbf{R}^{2}$ & Ad. & $\mathbf{f}^{2}$ & $\mathbf{Q}^{2}$ \\
\hline 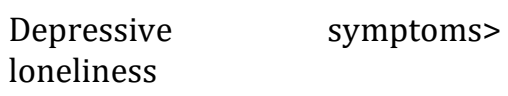 & 0.738 & $26.353^{* * *}$ & 0.000 & 0.545 & 0.542 & 1.198 & 0.188 \\
\hline $\begin{array}{l}\text { Depressive } \\
\text { pessimism }\end{array}$ & 0.598 & $10.720^{* * *}$ & 0.000 & 0.357 & 0.353 & 0.556 & 0.139 \\
\hline Pessimism> loneliness & 0.478 & $8.823^{* * *}$ & 0.000 & 0.229 & 0.223 & 0.296 & 0.074 \\
\hline
\end{tabular}

\subsection{Outcomes of MDCAT Score Impact on Different Symptoms}

The table 4 shows the impact of MDCAT scores on depressive symptoms, pessimism and loneliness. According to the above table correlation between MDCAT scores and depressive symptoms is assessed through path coefficient $=0.484$, whereas $\mathrm{t}$-value $=9.216$ and $\mathrm{Q}^{2}=0.074$ also shows there is a significant relationship between MDCAT scores and depressive symptom. Whereas the value of $\mathrm{R}^{2}$ coefficient $=0.235$ shows medium effect size of MDCAT on depressive symptom. According to the above table correlation between MDCAT scores and loneliness is assessed through path coefficient $=0.352$, whereas $\mathrm{t}$-value $=6.518$ and $\mathrm{Q}^{2}=0.037$ also shows there is a significant relationship between $\mathrm{MDCAT}$ scores and loneliness. Whereas the value of $\mathrm{R}^{2}$ coefficient $=0.124$ shows small effect. Similarly, according to the above table correlation between MDCAT scores and pessimism is assessed through path coefficient $=0.522$, whereas $\mathrm{t}$ value=9.131 and $\mathrm{Q}^{2}=0.102$ also shows there is a significant relationship between pessimism and MDCAT scores. Whereas the value of $\mathrm{R}^{2}$ coefficient $=0.272$ shows medium effect size of MDCAT scores on pessimism.

Table 4 Impact of MDCAT Scores on Pessimism, Depressive and loneliness symptoms

\begin{tabular}{|l|c|c|c|c|c|c|c|}
\hline \multicolumn{1}{|c|}{ Relationship } & $\begin{array}{c}\text { Path } \\
\text { Coefficient }\end{array}$ & t-value & $\begin{array}{c}\mathbf{p} \\
\text { value }\end{array}$ & $\mathbf{R}^{\mathbf{2}}$ & $\begin{array}{c}\text { Ad. } \\
\mathbf{R}^{\mathbf{2}}\end{array}$ & $\mathbf{f}^{\mathbf{2}}$ & $\mathbf{Q}^{\mathbf{2}}$ \\
\hline $\begin{array}{l}\text { MDCAT scores>Depressive } \\
\text { symptoms }\end{array}$ & 0.484 & $9.216^{* * *}$ & 0.000 & 0.235 & 0.229 & 0.306 & 0.074 \\
\hline MDCAT scores> loneliness & 0.352 & $6.518^{* * *}$ & 0.000 & 0.124 & 0.118 & 0.141 & 0.037 \\
\hline MDCAT scores> pessimism & 0.522 & $9.131^{* * *}$ & 0.000 & 0.272 & 0.267 & 0.374 & 0.102 \\
\hline
\end{tabular}

\subsection{Outcomes of Problem Focused Coping Role}

The table 5 shows the moderating role of problem focused coping on MDCAT scores and loneliness, which is not significant. According to the above table there is a significant negative correlation between problem focused coping and loneliness $(-0.414)$. Whereas the value of $\mathrm{R}^{2}$ coefficient $=0.276$ shows the medium effect size of problem focused coping on the loneliness.

Table 5 Moderating Effect of Problem Focused Coping on MDCAT Scores and Loneliness

\begin{tabular}{|l|c|c|c|c|c|c|c|}
\hline \multicolumn{1}{|c|}{ Relationship } & Path Coefficient & t-value & p value & $\mathbf{R}^{\mathbf{2}}$ & $\mathbf{A d}^{\mathbf{2}}$ & $\mathbf{f}^{\mathbf{2}}$ & $\mathbf{Q}^{\mathbf{2}}$ \\
\hline $\begin{array}{l}\text { MDCAT scores> } \\
\text { loneliness }\end{array}$ & 0.220 & $2.849^{* *}$ & 0.005 & - & - & 0.064 & - \\
\hline $\begin{array}{l}\text { Problem focused } \\
\text { Coping> loneliness }\end{array}$ & -0.061 & 0.942 & 0.347 & - & - & 0.006 & - \\
\hline $\begin{array}{l}\text { Problem focused } \\
\text { Coping> loneliness }\end{array}$ & -0.414 & $7.471^{* * *}$ & 0.000 & - & - & 0.217 & - \\
\hline & - & - & - & 0.276 & 0.261 & - & 0.092 \\
\hline
\end{tabular}




\subsection{Effect of Emotion Focused Coping}

The table 6 shows the moderating role of emotion focused coping on MDCAT scores and loneliness, which is not significant. According to the above table there is a positive correlation between emotion focused coping and loneliness $(0.545)$. Whereas the value of $R^{2}$ coefficient $=0.365$ shows the medium effect size of emotion focused coping on the loneliness.

Table 6 Moderating Effect of Emotion Focused Coping on MDCAT Scores and Loneliness

\begin{tabular}{|c|c|c|c|c|c|c|c|c|}
\hline \multicolumn{2}{|l|}{ Relationship } & Path Coefficient & $\begin{array}{l}\text { t- } \\
\text { value }\end{array}$ & p value & $\mathbf{R}^{2}$ & Ad. $\mathbf{R}^{2}$ & $\mathbf{f}^{2}$ & $\mathbf{Q}^{2}$ \\
\hline \multicolumn{2}{|c|}{ MDCAT scores> loneliness } & 0.097 & 1.288 & 0.198 & - & - & 0.012 & - \\
\hline $\begin{array}{l}\text { emotion focused } \\
\text { loneliness }\end{array}$ & Coping> & 0.104 & 0.870 & 0.385 & - & - & 0.017 & - \\
\hline $\begin{array}{l}\text { emotion focused } \\
\text { loneliness }\end{array}$ & Coping> & 0.545 & 0.954 & 0.340 & - & - & 0.373 & - \\
\hline Loneliness & & - & - & - & 0.365 & 0.352 & - & 0.124 \\
\hline
\end{tabular}

Table 7 Moderating Effect of Problem Focused Coping on MDCAT Scores and Pessimism

\begin{tabular}{|l|c|c|c|c|c|c|c|}
\hline Relationship & Path Coefficient & t-value & $\mathbf{p ~ v a l u e}$ & $\mathbf{R}^{\mathbf{2}}$ & Ad. $^{\mathbf{2}}$ & $\mathbf{f}^{\mathbf{2}}$ & $\mathbf{Q}^{\mathbf{2}}$ \\
\hline MDCAT scores> pessimism & 0.418 & $6.331^{* * *}$ & 0.000 & - & - & 0.232 & - \\
\hline $\begin{array}{l}\text { problem focused } \\
\text { coping> pessimism }\end{array}$ & -0.007 & 0.075 & 0.940 & - & - & 0.000 & - \\
\hline $\begin{array}{l}\text { problem focused } \\
\text { Coping> pessimism }\end{array}$ & -0.293 & $3.904^{* * *}$ & 0.000 & - & - & 0.106 & - \\
\hline Pessimism & - & - & - & 0.345 & 0.331 & - & 0.122 \\
\hline
\end{tabular}

Table 8 Moderating Effect of Emotion Focused Coping on MDCAT Scores and Pessimism

\begin{tabular}{|l|c|c|c|c|c|c|c|}
\hline Relationship & Path Coefficient & $\mathbf{t}$-value & $\mathbf{p}$ value & $\mathbf{R}^{\mathbf{2}}$ & $\mathbf{A d}^{\mathbf{2}} \mathbf{R}^{\mathbf{2}}$ & $\mathbf{f}^{\mathbf{2}}$ & $\mathbf{Q}^{\mathbf{2}}$ \\
\hline MDCAT scores> pessimism & 0.369 & $5.362^{* * *}$ & 0.000 & - & - & 0.181 & - \\
\hline $\begin{array}{l}\text { Emotion focused } \\
\text { coping> pessimism }\end{array}$ & -0.012 & 0.187 & 0.851 & - & - & 0.000 & - \\
\hline $\begin{array}{l}\text { Emotion focused } \\
\text { Coping> pessimism }\end{array}$ & -0.361 & 1.085 & 0.278 & - & - & 0.175 & - \\
\hline Pessimism & - & - & - & 0.370 & 0.357 & - & 0.134 \\
\hline
\end{tabular}

The table 7 shows the moderating role of problem focused coping on MDCAT scores and pessimism, which is not significant. According to the above table there is a significant negative correlation between problem focused coping and pessimism $(-0.293)$. Whereas the value of $\mathrm{R}^{2}$ coefficient $=0.345$ shows the medium effect size of problem focused coping on the pessimism. The table 8 shows the moderating role of emotion focused coping on MDCAT scores and pessimism, which is not significant. According to above table, there is significant positive relationship between MDCAT scores and pessimism (0.369). According to the above table there is a negative correlation between coping strategies and pessimism (-0.361). Whereas the value of $\mathrm{R}^{2}$ coefficient $=0.370$ shows the medium effect size of emotion focused coping on the pessimism. The table 9 shows the significant moderating role of problem focused coping on MDCAT scores and depressive symptom. According to the above table there is a significant negative correlation between problem focused 
coping and depressive symptoms (-0.197). Also, significant positive relationship between MDCAT scores and depressive symptoms (0.417). Whereas the value of $\mathrm{R}^{2}$ coefficient $=0.332$ shows the medium effect size of problem focused coping on the depressive symptoms. The table 10 shows the moderating role of emotion focused coping on MDCAT scores and depressive symptom, which is not significant. According to the above table there is a significant positive correlation between emotion focused coping and depressive symptoms (0.564). Also, significant positive relationship between MDCAT scores and depressive symptoms (0.237). Whereas the value of $R^{2}$ coefficient $=0.487$ shows the large effect size of emotion focused coping on the depressive symptoms.

Table 9 Moderating Effect of Problem Focused Coping on MDCAT Scores and Depressive Symptoms

\begin{tabular}{|l|c|c|c|c|c|c|c|}
\hline Relationship & Path Coefficient & $\mathbf{t}$-value & $\mathbf{p ~ v a l u e}$ & $\mathbf{R}^{\mathbf{2}}$ & $\mathbf{A d . ~}^{\mathbf{2}}$ & $\mathbf{f}^{\mathbf{2}}$ & $\mathbf{Q}^{\mathbf{2}}$ \\
\hline $\begin{array}{l}\text { MDCAT scores> depressive } \\
\text { symptoms }\end{array}$ & 0.417 & $6.417^{* * *}$ & 0.000 & - & - & 0.234 & - \\
\hline $\begin{array}{l}\text { Problem focused coping> } \\
\text { depressive symptoms }\end{array}$ & -0.204 & $2.274^{*}$ & 0.023 & - & - & 0.058 & - \\
\hline $\begin{array}{l}\text { Problem focused Coping> } \\
\text { depressive symptoms }\end{array}$ & -0.197 & $2.125^{*}$ & 0.034 & - & - & 0.047 & - \\
\hline Depressive symptoms & - & - & - & 0.332 & 0.318 & - & 0.104 \\
\hline
\end{tabular}

Table 10 Moderating Effect of Emotion Focused Coping on MDCAT Scores and Depressive Symptoms

\begin{tabular}{|l|l|c|c|c|c|c|c|}
\hline Relationship & $\begin{array}{l}\text { Path } \\
\text { Coefficient }\end{array}$ & t-value & $\begin{array}{l}\mathbf{p} \\
\text { value }\end{array}$ & $\mathbf{R}^{\mathbf{2}}$ & $\begin{array}{l}\text { Ad. } \\
\mathbf{R}^{\mathbf{2}}\end{array}$ & $\mathbf{f}^{\mathbf{2}}$ & $\mathbf{Q}^{\mathbf{2}}$ \\
\hline $\begin{array}{l}\text { MDCAT scores> depressive } \\
\text { symptoms focused coping> }\end{array}$ & 0.237 & $3.721^{* * *}$ & 0.000 & - & - & 0.087 & - \\
\hline $\begin{array}{l}\text { Emotion } \\
\text { depressive symptoms }\end{array}$ & 0.049 & 0.704 & 0.482 & - & - & 0.004 & - \\
\hline $\begin{array}{l}\text { Emotion focused Coping> } \\
\text { depressive symptoms }\end{array}$ & 0.564 & $5.556^{* * *}$ & 0.000 & - & - & 0.504 & - \\
\hline \begin{tabular}{l} 
Depressive symptoms \\
\hline
\end{tabular} & \multicolumn{2}{|l|}{-} & - & 0.487 & 0.476 & - & 0.159 \\
\hline
\end{tabular}

\subsection{Gender Difference}

Table 11 Independent Sample T-Test of Gender differences for Pessimism, Loneliness, Depressive Symptoms, Problem Focused Coping and Emotion Focused Coping

\begin{tabular}{|l|c|c|c|c|c|c|c|}
\hline \multicolumn{1}{|c|}{ Variables } & $\begin{array}{c}\text { Male } \\
(\mathbf{N = 6 2 )} \\
\mathbf{M ( S D )}\end{array}$ & $\begin{array}{c}\text { Female } \\
(\mathbf{N = 8 7 )}\end{array}$ & $\begin{array}{c}\text { Mean } \\
\text { Difference }\end{array}$ & $\boldsymbol{t}(\mathbf{S 1 4 7 )}$ & $\boldsymbol{P}$ & $\frac{\mathbf{9 5 \%}}{\boldsymbol{L L}}$ & $\underline{\boldsymbol{C l}}$ \\
\hline Pessimism & $7.57(3.45)$ & $7.7(3.91)$ & -.068 & -.109 & 0.913 & -1.30 & 1.16 \\
\hline Loneliness & $21.59(12.46)$ & $23.68(13.80)$ & -2.098 & -.952 & 0.343 & -6.46 & 2.26 \\
\hline Depressive Symptoms & $33.34(22.23)$ & $39.91(20.01)$ & -5.925 & -1.698 & 0.092 & -12.82 & 0.98 \\
\hline Problem focused coping & $23.86(5.26)$ & $25.44(5.56)$ & -1.59 & -1.753 & 0.082 & -3.37 & 0.21 \\
\hline Emotion focused coping & $39.12(7.28)$ & $41.46(6.07)$ & -2.35 & $-2.141 *$ & 0.034 & -4.52 & 0.19 \\
\hline
\end{tabular}

* Correlation is significant at the 0.05 level 
The results revealed that there were significant gender differences were found in emotional focused coping $(M=41.46$, $\mathrm{SD}=6.07$ ). Emotion focused coping were more in females than males (Table 11).

\section{Discussion}

The current research investigates how MDCAT scores effect the mental health of MDCAT failures. Current study explains the impact of MDCAT scores on pessimism, depressive and loneliness symptoms, furthermore this study also explain the moderating role of coping strategies between MDCAT scores and pessimism, depressive and loneliness symptoms. This study also aimed to find out the relationship between pessimism, depressive and loneliness symptoms. Moreover, study also explains the gender differences among these variables. The results suggest that there is a significant positive relationship between pessimism, depressive and loneliness symptoms which is consistent with other studies Study also found that there is significant impact of MDCAT scores on pessimism, depressive and loneliness symptoms, which is consistent with goal orientation model of depression, proposed by [12] which postulates that a performance orientation produces a susceptibility to depression, anxiety and negative affect through repeated failure. Many psychological problems like stress, anxiety and depression exist among $25 \%$ of students, at any given time. College students have many complex problems, like tough competition, new and hectic journey of studies, change in social life, pressure from parents to get excellent grades, feeling inferior by comparison with others, time pressure, fear of failure, greater academic demands and fear of parents that they would not accept them because he/she has undesirable result which make students anxious and depress all time, without any apparent reason [13].

According to Aaron Beck's Cognitive Theory of Depression, the more pessimist or negative thinking a person is, the more he will depress [14]. Beck describe negative Cognitive Triad, which is if a person think he is incompetent or inadequate secondly, he thought that his experience result in failure and thirdly he is hopeless about future then he surely will have depression. Likewise, researcher found in MDCAT failures that they have pessimist view of self and about future. These students have set goals of life and low academic achievement in MDCAT leads to pessimism and depression. The theory of Conditional Goal Setting (CGS), give new explanation for the relationship between depression and goals. This theory focus on why individuals choose and pursue goal. This theory suggest that abstract goals or higher order goals are less likely to achieve and leads to depression [15].

Results also suggest that problem focused coping has significant negative relationship with depressive symptoms, loneliness and pessimism. Emotion focused coping has negative relationship with pessimism and positive with loneliness. Which is consistent with previous studies which also suggested that loneliness has significant negative correlation with problem focused and positive with emotion focused coping. Furthermore, emotion focused coping has significant positive relationship with depressive symptoms; similar findings are given by previous studies $[16,17]$. Additionally, moderating role of emotion focused coping is not significant between MDCAT scores and depressive symptoms, loneliness and pessimism among MDCAT failures. Moreover, problem focused coping significantly moderate the relationship between MDCAT score and depressive symptoms, similar results were found previously that Coping responses as moderators of the impact of stressful events on depressive symptom. According to The transactional theory of stress and coping that if a person possesses good coping skills than stressor has little impact on him and if coping is ineffective than may have damaging results for person's psychological health and social functioning [18]. Although unsuccessful adaptation of skills leads to initiate further more skills and continued failure leads to negative effect. In support of this theory researcher found that MDCAT failures possesses ineffective coping skills and facing psychological problems (depressive symptoms, loneliness and pessimism) moreover disturbance in social functioning [19]. Moreover, current study found that females use more emotion focused coping, which is consistent with other studies. It was also found previously that females use more emotion focused coping and less problem focused coping because of high scores on depressive symptoms.

\section{Conclusion}

MDCAT failures possess pessimism, depressive and loneliness symptoms. Additionally, depressive symptoms, loneliness and pessimism are interlinked. Furthermore, MDCAT failures use less problem focused coping and possess more depressive symptoms, loneliness and have pessimism because problem focused coping has significant negative relation with all these. Problem focused coping also significantly moderate the relation between MDCAT scores and depressive symptoms. Overall, females were found having more emotion focused coping.

\section{Limitations and Suggestions}

Purposive sampling technique was used for study. Only MDCAT failures were selected as sample. Sample size was small and study conducted in Bahawalpur City so that's why cannot generalize the results to all population. In future, income 
of parents and parents support / social support should be checked. Sample should be selected from all cities of Punjab. Sample size should be maximizing. Proper coaching and psychological counseling should be given to the failed students and impact of this should be checked.

\section{Compliance with ethical standards}

\section{Acknowledgments}

It is acknowledged that the credit of this research goes to Professor Doctor Masood Nadeem who supported whole time.

\section{Disclosure of conflict of interest}

There is no conflict of interests to declare from anyone of authors.

\section{Statement of informed consent}

It is notified that informed consent was obtained from all individual participants included in the study.

\section{References}

[1] Hill MR, Goicochea S, Merlo LJ. In their own words: stressors facing medical students in the millennial generation. Med Educ Online. 2018; 23(1): 1530558.

[2] Al Saadi T, Addeen SZ, Turk T, Abbas F, Alkhatib M. Psychological distress among medical students in conflicts: a cross-sectional study from Syria. BMC Med Educ. 2017; 17(1): 1-8.

[3] Zhang MWB, Lim RBC, Lee C, Ho RCM. Prevalence of internet addiction in medical students: a meta-analysis. Acad Psychiatry. 2018; 42(1): 88-93.

[4] Yücens B, Üzer A. The relationship between internet addiction, social anxiety, impulsivity, self-esteem, and depression in a sample of Turkish undergraduate medical students. Psychiatry Res. 2018; 267: 313-8.

[5] Pacheco JP, Giacomin HT, Tam WW, Ribeiro TB, Arab C, Bezerra IM, et al. Mental health problems among medical students in Brazil: a systematic review and meta-analysis. Brazilian J Psychiatry. 2017; 39: 369-78.

[6] Adhikari A, Dutta A, Sapkota S, Chapagain A, Aryal A, Pradhan A. Prevalence of poor mental health among medical students in Nepal: a cross-sectional study. BMC Med Educ. 2017; 17(1): 1-7.

[7] Frajerman A, Morvan Y, Krebs MO, Gorwood P, Chaumette B. Burnout in medical students before residency: a systematic review and meta-analysis. Eur Psychiatry. 2019; 55: 36-42.

[8] Zeng W, Chen R, Wang X, Zhang Q, Deng W. Prevalence of mental health problems among medical students in China: A meta-analysis. Medicine (Baltimore). 2019; 98(18).

[9] Wolf MR, Rosenstock JB. Inadequate sleep and exercise associated with burnout and depression among medical students. Acad psychiatry. 2017; 41(2): 174-9.

[10] Mahroon ZA, Borgan SM, Kamel C, Maddison W, Royston M, Donnellan C. Factors associated with depression and anxiety symptoms among medical students in Bahrain. Acad Psychiatry. 2018; 42(1): 31-40.

[11] Bilal A, Riaz A. Academic stress and suicidal ideation in MDCAT repeating candidates: mediating role of depression and moderating role of age and gender. Pakistan J Physiol. 2020; 16(3): 7-10.

[12] Al-Alawi M, Al-Sinawi H, Al-Qubtan A, Al-Lawati J, Al-Habsi A, Al-Shuraiqi M, et al. Prevalence and determinants of burnout syndrome and depression among medical students at Sultan Qaboos University: a cross-sectional analytical study from Oman. Arch Environ Occup Health. 2019; 74(3): 130-9.

[13] Tian-Ci Quek T, Tam W-S, X Tran B, Zhang M, Zhang Z, Su-Hui Ho C, et al. The global prevalence of anxiety among medical students: a meta-analysis. Int J Environ Res Public Health. 2019; 16(15): 27-35.

[14] Silva V, Costa P, Pereira I, Faria R, Salgueira AP, Costa MJ, et al. Depression in medical students: insights from a longitudinal study. BMC Med Educ. 2017; 17(1): 1-9.

[15] Fawzy M, Hamed SA. Prevalence of psychological stress, depression and anxiety among medical students in Egypt. Psychiatry Res. 2017; 255: 186-94. 
[16] Azad N, Shahid A, Abbas N, Shaheen A, Munir N. Anxiety and depression in medical students of a private medical college. J Ayub Med Coll Abbottabad. 2017; 29(1): 123-7.

[17] Mao Y, Zhang N, Liu J, Zhu B, He R, Wang X. A systematic review of depression and anxiety in medical students in China. BMC Med Educ. 2019; 19(1): 1-13.

[18] Ngasa SN, Sama C-B, Dzekem BS, Nforchu KN, Tindong M, Aroke D, et al. Prevalence and factors associated with depression among medical students in Cameroon: a cross-sectional study. BMC Psychiatry. 2017; 17(1): 1-7.

[19] Moir F, Yielder J, Sanson J, Chen Y. Depression in medical students: current insights. Adv Med Educ Pract. 2018; 9: 323. 